\title{
Indirect search for neutralinos at neutrino telescopes
}

\author{
A. Bottino, V. de Alfaro, N. Fornengo, G. Mignola and M. Pignone \\ Dipartimento di Fisica Teorica, Ĺniversità di Torino, and INFN, Sezione di Torino, Via P. Giuria I, 1-10125 Turin, Italy
}

Received 2 May 1991

\begin{abstract}
Under the assumption that cold dark matter is composed (at least partially) of neutralinos, capture and pair annihilation of these particles in the sun and the earth are evaluated in the framework of the minimal SUSY extension of the standard model (MSSM), taking into account the effects due to the radiative corrections to the masses of the Higgs bosons. The flux of the neutrinos produced in the neutralino-neutralino annihilation and the relevant rate of the muons generated by neutrino conversion in the earth and crossing a detector upwardly are calculated. The present experimental limits from underground detectors are employed to set constraints on the MSSM parameters, and the capabilities of future neutrino telescopes to further explore regions in parameter space are analyzed.
\end{abstract}

Recent astrophysical observations confirm the existence of invisible mass in our universe and indicate that this dark matter may be due to more than one single candidate.

More specifically, on the one side a thorough analysis about the local brightness of spiral galaxies [1] has disproved the claim put forward recently [2] according to which opacity features in these galaxies would undermine the evidence for dark matter from rotation curves. It then follows that the existence of invisible matter appears to be an unescapable consequence of astrophysical observational facts.

On the other hand, the most recent astrophysical observations by infrared measurements (IRAS) [3] about very remote regions of the universe (up to distances of about $140 h^{-1} \mathrm{Mpc} ; h$ is the normalized Hubble parameter) indicate that the nature of the dark matter may be even more complicated than it was believed before. In fact, the presence of structures over very different scales may suggest that there could be a conspiracy of different sorts of particles constituting the dark matter, each kind contributing a fraction of the density; possibly cold dark matter components, necessary to explain galaxies and galaxy clusters, have to coexist with hot dark matter candidates to account for the large-scale $\left(20 h^{-1} \mathrm{Mpc}\right)$ structures evidenced by the IRAS measurements.

This situation requires more detailed investigation about the possible particle candidates for dark matter and their experimental detection. In the present paper we address the problem of cold dark matter in the light of the recent theoretical developments in supersymmetric models and of all the available constraints due to experiments at accelerators and at underground detectors as well.

As it was considered by many authors $"$, a very appealing candidate for dark matter is supplied by the neutralino, provided this particle occurs to be the lightest supersymmetric particle (LSP) and $R$-parity is conserved ${ }^{\# 2}$. Extensive work has been devoted recently to the investigations about different means for detecting this particle, if it really constitutes a sizeable component of dark matter in the universe. Direct detection through neutralino-nucleus scattering [49] appears to require an instrumental sensitivity which is very hard to achieve with the present experimental set-ups. A more promising approach is an indirect search based on the detection of signals related to pair annihilation of neutralinos inside celestial bodies (either sun or earth ) [10-18]. This latter approach is the main subject of the present analysis. We

*1 The detection of neutralinos either by direct or by indirect methods were discussed respectively in refs. [4-9] and refs. [10-18] and in the references therein. See also ref. [19] for a thorough review in the field.

*2 As general reviews on supersymmetric models see ref. [20]. 
do not discuss here another possibility for indirect detection of dark matter, namely the observation of $\chi-\chi$ annihilations in the galactic halo via detection of $\overline{\mathrm{p}}, \mathrm{e}^{+}$(due to $\mathrm{q}-\overline{\mathrm{q}}$ and $\mathrm{\ell}-\bar{\ell}$ production) or detection of the gamma line at $E_{\gamma}=m_{\chi}$. These processes have been discussed by a number of authors [21].

In this paper, after a short outline of the theoretical scheme employed, we discuss the neutralino relic abundance, the capture of neutralinos in the sun and in the earth and the neutrino outputs generated by the pair annihilation of the neutralinos trapped in the celestial bodies. Finally, we report our results on the flux of the muons which are produced by neutrinomuon conversion in the earth and which would cross a detector upwardly. Our evaluations of the up-going muon signals are then employed to convert present experimental limits into exclusion plots in the space of the parameters used in the present analysis and to establish the capabilities of investigation in this field by the future neutrino telescopes.

Our theoretical framework is the minimal supersymmetric extension of the standard model (MSSM) [20]. In this scheme the Majorana fermions which are SUSY partners of the neutral bosons are: two gauginos $\tilde{W}_{3}, \widetilde{B}$ and two higgsinos $\tilde{\mathrm{H}}_{1}, \tilde{\mathrm{H}}_{2}$; neutralinos, $\chi$, are defined as linear combinations thereof.

Using the standard definition of the photino, $\tilde{\gamma}$, and the zino, $\tilde{Z}$, states

$\bar{\gamma}=\cos \theta_{\mathrm{w}} \widetilde{\mathbf{B}}+\sin \theta_{\mathrm{w}} \tilde{\mathrm{W}}_{3}$,

$\tilde{\mathrm{Z}}=-\sin \theta_{\mathrm{w}} \tilde{\mathrm{B}}+\cos \theta_{\mathrm{w}} \tilde{\mathrm{W}}_{3}$,

where $\theta_{w}$ is the Weinberg angle, the $\chi$ states are explicitly written as

$\chi=a_{1} \tilde{\gamma}+a_{2} \tilde{\mathrm{Z}}+a_{3} \tilde{\mathrm{H}}_{1}^{0}+a_{4} \widetilde{\mathrm{H}}_{2}^{0}$.

In the following we will refer to the neutralino as the particlc of lowest mass among those defined by eq. (2). This will be assumed to be the LSP, to be stable (due to $R$-parity conservation) and then to have the necessary prerequisites to be considered as a candidate for dark matter. In the MSSM the independent parameters are only a few: a $\widetilde{B}$ mass paramcter $M_{1}$, a $\tilde{W}_{3}$ mass parameter $M_{2}$, a Higgs mixing mass parameter $\mu$ and $\tan \beta=v_{2} / v_{1} \quad\left(v_{1}\right.$ and $v_{2}$ being the Higgs VEVs giving masses to the down-type and up-type quarks, respectively). We also assume the usual relationship $M_{1}=\frac{5}{3} M_{2} \tan ^{2} \theta_{w}$ implied by the embedding of the MSSM in a grand unification scheme.

Furthermore in the MSSM there are three neutral Higgs particles: two $C P$-even scalars, denoted here by $\mathrm{h}$ and $\mathrm{H}$, and one $C P$-odd scalar $\mathrm{A}$. If the Higgs potential is treated at the tree level, then the well known mass inequalities

$m_{\mathrm{h}} \leqslant m_{\mathrm{A}} \leqslant m_{\mathrm{H}}$,

$m_{\mathrm{h}} \leqslant|\cos 2 \beta| m_{\mathrm{Z}}$

follow and the Higgs phenomenology depends on two parameters only: $\tan \beta$ and one Higgs mass. One of the major recent breakthroughs in the supersymmetric models has been the recognition that radiative corrections modify the tree-level mass properties of the Higgs particles very significantly [22]. In fact it can be shown that, under rather general assumptions, the following mass relationship holds:

$m_{\mathrm{h}, H}=\frac{1}{2}\left(m_{\mathrm{A}}^{2}+M_{2}^{2}+\epsilon \pm \Delta\right)$,

where $A$ is given by

$$
\begin{gathered}
\Delta=\left[\left(m_{\mathrm{A}}^{2}+M_{Z}^{2}+\epsilon\right)^{2}-4 m_{\mathrm{A}}^{2} \cdot M_{Z}^{2} \cos ^{2} 2 \beta\right. \\
\left.-4 \epsilon m_{\mathrm{A}}^{2} \sin ^{2} \beta-4 \epsilon M_{Z}^{2} \cos ^{2} \beta\right]^{-1 / 2,}
\end{gathered}
$$

and $\epsilon$ represents the one-loop radiative corrections due to the top quark and its scalar SUSY partners

$\epsilon=\frac{3 \alpha_{\mathrm{W}} m_{\mathrm{t}}^{4}}{2 \pi M_{\mathrm{W}}^{2} \sin ^{2} \beta} \log \left(1+\frac{m^{2}}{m_{\mathrm{\imath}}^{2}}\right)$.

Here $m_{1}$ and $m$ denote the top mass and the topscalar mass, respectively; $\alpha_{w}$ is the $\mathrm{SU}(2)$ fine structure constant. It is important to notice that eqs. (5)(7) invalidate the tree-level inequalities (eqs. (3), (4) ). It is easy recognized that the one-loop radiative corrections have the general feature of increasing the tree-level mass values by an amount which depends on the top mass very strongly. In particular this makes the mass differences, c.g. $m_{\mathrm{A}}-m_{\mathrm{h}}$, to depend sensitively on the radiative contributions, especially at low values for $\tan \beta$. These properties turn out to be very relevant for the discussions to be developed later.

We turn now to the problem of the indirect detection of neutralinos, under the assumption that these are (at least, partial) constituents of the dark matter. The first relevant quantity to be evaluated is the neutralino relic abundance $\Omega h^{2}$ ( $\Omega$ being the ratio of the neutralino matter density to the critical density). This 
quantity has been calculated here by the method discussed in ref. [23]; the expression is

$$
\begin{gathered}
\Omega h^{2}=2.13 \times 10^{-11}\left(\frac{T_{\chi}}{T_{\gamma}}\right)^{3}\left(\frac{T_{y}}{2.7 \mathrm{~K}}\right)^{3} \\
\times N_{\mathrm{F}}^{1 / 2}\left(\frac{\mathrm{GeV}^{-2}}{a x_{\mathrm{f}}+\frac{1}{2} b x_{\mathrm{f}}^{2}}\right) \mathrm{g} \mathrm{cm}^{-3},
\end{gathered}
$$

where $x_{\mathrm{f}}=T_{\mathrm{f}} / m_{\mathrm{x}}$ ( $T_{\mathrm{f}}$ is the neutralino freeze-out temperature, $m_{\chi}$ is the neutralino mass $), T_{\gamma}$ is the present temperature of the microwave background, $T_{\chi} / T_{\gamma}$ is the reheating factor for the photon temperature as compared to the neutralino temperature, $N_{\mathrm{f}}$ is the effective number of relativistic degrees of freedom at $T_{\mathrm{f}}, a$ and $b$ are the parameters which enter in the lowtemperature expansion for the thermally-averaged product of the pair-annihilation cross section times the relative velocity

$\langle\sigma v\rangle=a+b x_{\mathrm{f}}$.

For the evaluation of $\langle\sigma v\rangle$ we have taken into account the $\chi-\chi$ annihilation processes into $f-f$ pairs and into Higgs pairs by exchange of $Z^{0}$ and of Higgses in the $s$-channel and by exchange of sfermions and neutralinos in the $t$-channel. (In the present analysis only neutralinos with $m_{\chi}$ less than the $\mathrm{W}$-mass have been considered.) These processes (or only some of them) have been considered in analyses by various authors [18,23-26]. Differently from previous papers, oneloop corrected relationships between the Higgs masses (eqs. (5)-(7)) have been employed here. This is relevant for processes with exchange of Higgs particles, since these depend sensitively on the kinematical positions of the pole singularities in the s-channel. Here we consider values of $m_{\mathrm{h}}$ larger than, or equal to, 50 $\mathrm{GeV}$ (actually the present experimental lower bound for $m_{\mathrm{h}}$ is $38-48 \mathrm{GeV}$ depending on the value of $\tan \beta$ ) [27].

The results of our evaluations of $\Omega h^{2}$ show that in large regions of our parameter space the neutralino relic abundance is much less than 0.01 ; if we take into account the numerical range for $h(0.4<h<1)$, we conclude that in these parameter regions neutralinos cannot be the only component of dark matter in the halos of galaxies, since there $\Omega$ is less than 0.05 . Similar conclusions were obtained in previous papers (e.g. in ref. [18]); but relevant regions are now shifted in the parameter space, due to the new mass relations between the Higgs particles. As expected, the dependence on this effect is significant, especially at small $\tan \beta$ values. To illustrate this point, we report in fig. 1 our results for $m_{\mathrm{h}}=50 \mathrm{GeV}$ and $\tan \beta=2$. Here one notes, e.g., in the up-right $M_{2}-\mu$ region that the minima of $\Omega h^{2}$ correspond to the pole in $\sigma$ around $m_{\mathrm{x}} \simeq \frac{1}{2} m_{\mathrm{A}}$, which at fixed $m_{\mathrm{h}}$ is displaced according to the radiative formulas (5) as a function of $m_{1}$.

The capture rate $C$ of the neutralinos by macroscopical bodies has been evaluated as in refs. $[28,29]$. For other evaluations see ref. [30]. For the sake of the present discussion $C$ may be schematically reported as follows $[17,18]$ :

$C=\frac{\rho_{\chi}}{v_{\chi}} \sum_{i} \frac{\sigma_{t}}{m_{\chi} m_{t}}\left(M_{\mathrm{B}} f_{l}\right)\left\langle v_{\mathrm{esc}}^{2}\right\rangle, X_{l}$,

where $\rho_{\chi}$ and $v_{\chi}$ are the neutralino density and mean velocity, $\sigma_{t}$ is the cross section of the neutralino with the nucleus $i$ of mass $m_{i} . M_{\mathrm{B}} f_{i}$ is the total mass of the element $i$ in the body of mass $M_{\mathrm{B}},\left\langle v_{\mathrm{csc}}^{2}\right\rangle_{i}$ is the square escape velocity averaged over the distribution of the element $i ; X$, is a factor which takes account of kinematical properties occurring in the neutralinonucleus interactions.

In what follows $v_{x}$ has been taken as $v_{x}=300 \mathrm{~km} \mathrm{~s}^{-1}$. For $\rho_{x}$ the value $\rho_{x}=0.3 \mathrm{GeV} \mathrm{cm} \mathrm{cm}^{-3}$ has been used whenever our values for $\Omega h^{2}$ are larger than 0.05 ; otherwise that value for $\rho_{\chi}$ has been scaled by the factor $\Omega h^{2} / 0.05$ (we are following here the same strategy as in ref. [ 18$]$.

We anticipate that the final $\mu$ yicld due to the capture rate in the earth is much higher than that due to the sun (roughly by a factor $10-100$ ). This is due to the larger coherent capture cross section which is dramatically enhanced by the couplings of the Higgs particles to the sea of the strange quarks as compared to the non-strange quarks, as evaluated in refs. [31,32] and applied to the present problem in ref. [18].

The neutralinos, accumulated in the celestial bodies, undergo annihilation processes

$\chi+\chi \rightarrow \mathrm{f}+\overline{\mathrm{f}}$.

$\chi+\chi \rightarrow h+A \rightarrow f+\bar{f}+X$,

via the diagrams mentioned above. Both reactions in eq. (11) generatc a neutrino signal whose differential spectrum at a distance $d$ from the annihilation region may be written as 

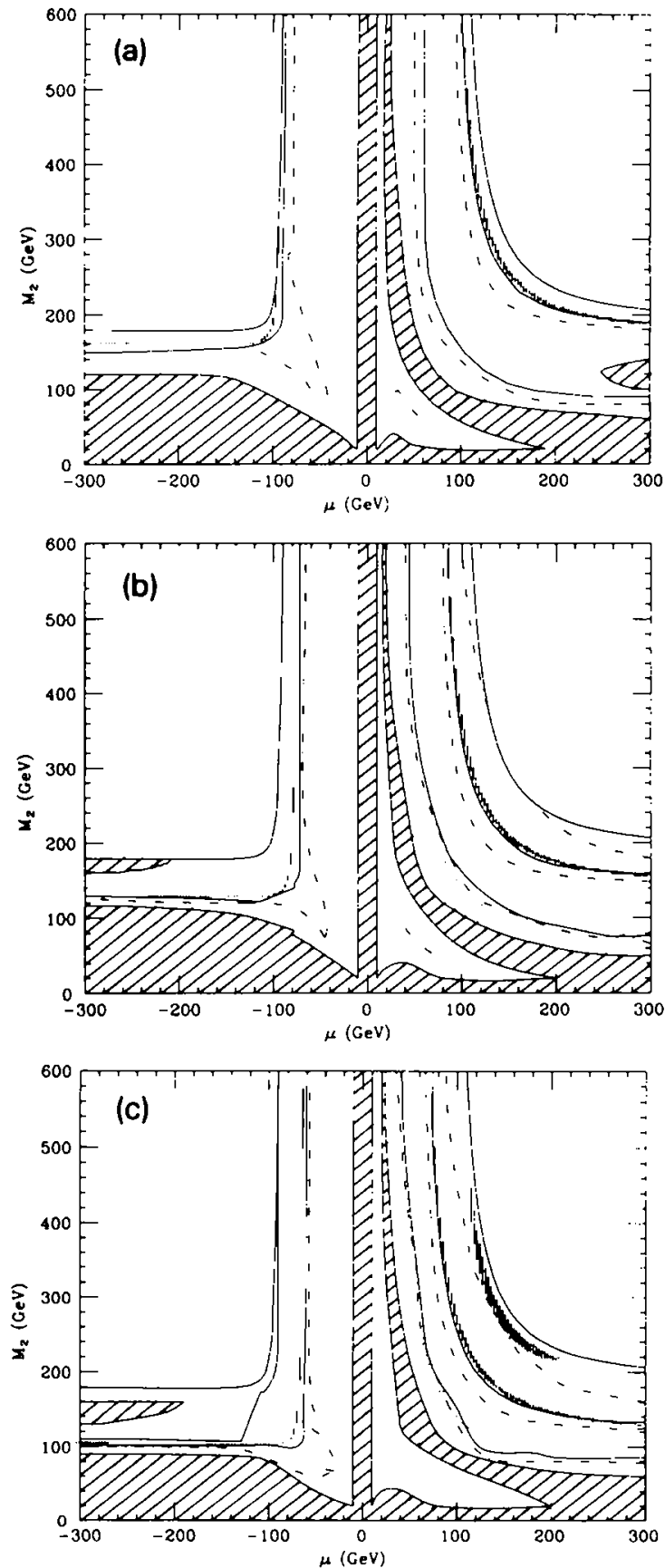

Fig. 1. Neutralino relic abundance $\Omega h^{2}$ in the $M_{2}-\mu$ parameter space for $\tan \beta=2$ and $m_{\mathrm{h}}=50 \mathrm{GeV}$. The figures refer to the following cases: (a) no radiative corrections; (b) radiative corrections with $m_{1}=100 \mathrm{GeV}$; (c) radiative corrections with $m_{1}=130$ GeV. Dash-dotted lines are lines where $\Omega h^{2}=0.05$, solid lines are lines where $\Omega h^{2}=0.01$, dotted regions are regions where $\Omega h^{2} \leqslant 0.001$ : hatched domains are regions where overclosure occurs. $\frac{\mathrm{d} N_{v}}{\mathrm{~d} E_{\mathrm{v}}}=\frac{R_{\mathrm{a}}}{4 \pi d^{2}} \sum_{\mathrm{f}} B_{\mathrm{f}} \frac{\mathrm{d} N_{\mathrm{f}, \mathrm{v}}}{\mathrm{d} E_{\mathrm{v}}}$,

where $R_{\mathrm{a}}$ is the $\chi \chi$ annihilation rate, $B_{\mathrm{f}}$ is the branching ratio for the production of the fermion $\mathrm{f}$ and $\mathrm{d} N_{\mathrm{f}, \mathrm{v}} /$ $\mathrm{d} E_{\mathrm{v}}$ is the differential distribution for the neutrino yield in the fermion semileptonic decay; in eq. (12) a sum over channels is understood. By making the usual assumption that at the present epoch cquilibrium between neutralino capture and annihilation is attained, $R_{\mathrm{a}}=\frac{1}{2}$ C' is replaced in eq. (12).

For the evaluation of the neutrino yield $\mathrm{d} N_{\mathrm{f}, \mathrm{v}} / \mathrm{d} E_{\mathrm{v}}$ we have employed the numerical simulations of ref. [16], where a Lund Monte Carlo is used to calculate the neutrino spectra due to the decay of $f-f$ pairs produced in annihilation processes. The specific process considered in ref. [16] is $\mathrm{e}^{+} \mathrm{e}^{-}$annihilation; fragmentation properties are properly taken into account in the Monte Carlo and corrections due to the influence of the medium on the final states are also included. We write the neutrino distribution in the usual way

$\frac{\mathrm{d} N_{\mathrm{f}, v}}{\mathrm{~d} E_{\mathrm{v}}}=\frac{1}{E_{\mathrm{f}}} \int_{E_{\mathrm{v}} / E_{\mathrm{r}}}^{\mathrm{I}} \mathrm{d} y \frac{1}{y} \frac{\mathrm{d} N}{\mathrm{~d} y}$,

where $\mathrm{d} N / \mathrm{d} y$ is parametrized as

$\frac{\mathrm{d} N}{\mathrm{~d} y}=\sum_{n=1}^{4} a_{n} y^{n}$

$E_{\mathrm{f}}$ is the fermion encrgy if $\mathrm{f}$ is a lepton, but, in case $\mathrm{f}$ is a quark, $E_{\mathrm{f}}$ is the average energy of the hadrons generated by that quark; $y$ is given by $y=2 E_{\mathrm{v}} / m_{\mathrm{f}}$, where $m_{\mathrm{f}}$ is the fermion mass. The $\mathrm{f}-\mathrm{f}$ pairs included in the analysis are those which are relevant for the production of the neutrino spectrum: $\tau \bar{\tau}, c \bar{c}, b \bar{b}$ (from now on by neutrino spectrum we actually mean the sum of the $v_{\mu}$ and $\bar{v}_{\mu}$ spectra ).

We have determined the coefficients $a_{n}$ by using directly the moments of the original Monte Carlo distribution of ref. [16]. Our results are reported in table 1 ; they differ from the ones given in ref. [16] and other references $[17,18]$ and do not generate unphysical features in the $\mathrm{d} N / \mathrm{d} y$ distributions.

Given the differential neutrino flux in eq. (12) on the earth surface, the differential flux of the muons which are generated by neutrino conversion in the 
Table 1

Values of the coefficients $a_{n}$ occurring in eq. (14) for lepton $\tau$ and quarks $c$ and $b$.

\begin{tabular}{lrrrr}
\hline Parent & \multicolumn{1}{l}{$a_{1}$} & \multicolumn{1}{l}{$a_{2}$} & \multicolumn{1}{l}{$a_{3}$} & \multicolumn{1}{l}{$a_{4}$} \\
\hline$\tau \bar{\tau}$ & -0.0484 & 2.4082 & -2.6158 & 0.2422 \\
$\mathrm{c} \overline{\mathrm{c}}$ & -0.2732 & 4.1969 & -7.8196 & 3.8976 \\
$\mathrm{~b} \overline{\mathrm{b}}$ & 4.4711 & -14.665 & 16.969 & -6.8376 \\
\hline
\end{tabular}

earth and then cross a detector upwardly is provided by the formula [33]

$\frac{\mathrm{d} N_{\mu}}{\mathrm{d} E_{\mathrm{v}}}=N_{\mathrm{A}} \frac{\mathrm{d} N_{\mathrm{v}}}{\mathrm{d} E_{\mathrm{v}}} \int_{\varepsilon_{\mu, \mathrm{th}}}^{E_{\mathrm{v}}} \frac{\mathrm{d} \sigma\left(E_{\mathrm{v}}, E_{\mu}^{\prime}\right)}{\mathrm{d} E_{\mu}^{\prime}} R\left(E_{\mu}^{\prime}, E_{\mu, \mathrm{th}}\right)$,

where $R\left(E_{\mu}^{\prime}, E_{\mu}\right)$ is the mean range in rock for a muon with initial and final energies $E_{\mu}^{\prime}, E_{\mu}$, respectively, $E_{\mu, t h}$ is the muon energy threshold and $N_{\mathrm{A}}$ is the Avogadro number. The average range $R$ has been evaluated with a Monte Carlo (GEANT) code, which includes the following energy-loss processes: ionization, pair production, bremsstrahlung and photonuclear effects. If the average energy loss is written, as usual, in terms of two constant parameters $A$ and $B$

$-\frac{\mathrm{d} E}{\mathrm{~d} x}=A+B E$,

then the range is given by

$R\left(E_{\mu}^{\prime}, E_{\mu}\right)=\frac{1}{B} \ln \frac{1+(B / A) E_{\mu}^{\prime}}{1+(B / A) E_{\mu}}$,

and the up-going muon spectrum in the $E_{\mu}$ variable may be written as

$$
\begin{aligned}
& \frac{\mathrm{d} N_{\mu}}{\mathrm{d} E_{\mu}}=N_{\mathrm{A}} \frac{1}{A\left[1+(B / A) E_{\mu}\right]} \int_{E_{\mu, \mathrm{th}}}^{\infty} \mathrm{d} E_{\mathrm{v}} \frac{\mathrm{d} N_{\mathrm{v}}}{\mathrm{d} E_{v}} \\
& \quad \times \int_{E_{\mu}}^{E_{v}} \mathrm{~d} E_{\mu}^{\prime} \frac{\mathrm{d} \sigma\left(E_{\mathrm{v}}, E_{\mu}^{\prime}\right)}{\mathrm{d} E_{\mu}^{\prime}} .
\end{aligned}
$$

A best fit to our Monte Carlo results provides the following values for $A$ and $B$ :

$$
\begin{aligned}
& A=2.4 \times 10^{-3} \mathrm{GeV} /\left(\mathrm{g} / \mathrm{cm}^{3}\right), \\
& B=4.75 \times 10^{-6}\left(\mathrm{~g} / \mathrm{cm}^{2}\right)^{-1} .
\end{aligned}
$$

These values are larger, by approximately $20 \%$, than the relevant values in ref. [33], the ratio being essentially the same (in ref. [33] energy loss by pair pro- duction has been neglected). Our values are comparable with those reported in ref. [34] (in this reference $A$ and $B$ are taken explicitly energy dependent) $[35]^{\sharp 3}$. For the differential cross section $\mathrm{d} \sigma\left(E_{v}, E_{\mu}^{\prime}\right) / \mathrm{d} E_{\mu}^{\prime}$ we have employed $\left(z=1-E_{\mu}^{\prime} / E_{v}\right)$

$$
\begin{aligned}
& \frac{\mathrm{d} \sigma_{\mathrm{v}(\overline{\mathrm{v}})}}{\mathrm{d} E_{\mu}^{\prime}}=\frac{G_{\mathrm{F}}^{2} m}{\pi} \int_{0}^{1} \mathrm{~d} x \frac{M_{\mathrm{W}}^{4}}{\left(2 m x z E_{\mathrm{v}}+M_{\mathrm{W}}^{2}\right)^{2}} \\
& \quad \times\left[\left(1-z+\frac{1}{2} z^{2}\right) F_{2}(x) \pm\left(z-\frac{1}{2} z^{2}\right) x F_{3}(x)\right],
\end{aligned}
$$

with the following parametrizations for the structure functions [36]:

$$
\begin{aligned}
& F_{2}(x)=3.9 x^{0.55}(1-x)^{3 / 2}+1.1(1-x)^{8}, \\
& x F_{3}(x)=3.6 x^{0.55}(1-x)^{3.2} .
\end{aligned}
$$

Also the background of the up-going muons due to atmospheric neutrinos has been evaluated by using the previous formulae (15)-(21). This background is assumed to be given by a power-law neutrino spectrum with a spectral index equal to 3.2 ; the background muon spectrum is normalized to a rate of $2 \times 10^{-13} \mathrm{~cm}^{-2} \mathrm{~s}^{-1} \mathrm{sr}^{-1}$ for upward muons with energy larger than $2 \mathrm{GeV}[33,37]$.

Our analysis on the muon rates due to $\chi-\chi$ annihilation in the earth and in the sun has been carried out by varying the MSSM parameters in their full parameter space and for the allowed range of $m_{\mathrm{h}}$. As an example of our results, in fig. 2 we report the signals of the upgoing muons due to the capture in the earth, for $m_{\mathrm{h}}=80 \mathrm{GeV}$ and $\tan \beta=8$. In the $M_{2}-\mu$ parameter space, exclusion regions due to experimental upper limits on the up-going muon yield are reproduced. Of the various experimental results at underground detectors $[38,40]$ we consider the ones from Kamioka [38] which provide the most stringent constraints on our parameters. These refer to an exposure of $759 \mathrm{~m}^{2} \mathrm{yr}$ (area of $155 \mathrm{~m}^{2}$, running time $4.9 \mathrm{yr}), 2 \mathrm{GeV}$ muon energy threshold, with a total number of events, within a cone of $30^{\circ}$ half-aperture (around the earth center) equal to 26 , the same as the expected number for the background. Using our evaluations for the muon rates, we exclude (at $95 \%$ $\mathrm{CL}$ ) the regions marked by stars in the $M_{2}-\mu$ param-

*3 For evaluations of the energy loss see also the Particle Data Group [35]. 

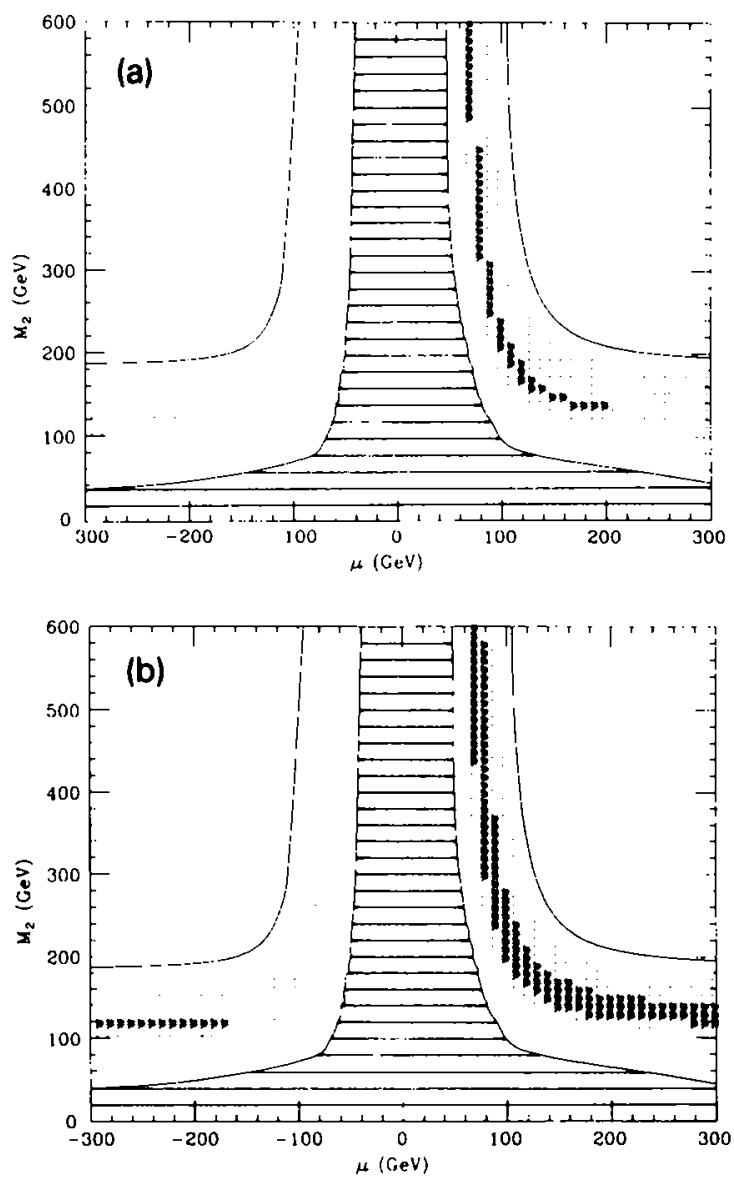

Fig. 2. Signals of up-going muons in the $\boldsymbol{H}_{2}-\mu$ parameter space for $\tan \beta=8$. The figures refer to $m_{\mathrm{h}}=80 \mathrm{GeV}$ : (a) radiative corrections with $m_{1}=100 \mathrm{GeV}$; (b) radiative corrections with $m_{1}=160 \mathrm{GeV}$. The regions denoted by stars are excluded (at $95 \%$ CL) by the experimental results of ref. [38]; the dotted regions are regions where the signal would produce a 4- $\sigma$ effect in a neutrino telescope of size $10^{5} \mathrm{~m}^{2}$ in two years (muon threshold energy of $2 \mathrm{GeV}$ ). The hatched region is excluded by LEP [ 39].

eter space. We notice that these regions roughly correspond to the level of sensitivity of MACRO in one year of running at full scale. Some words of warning have to be said in connection with the exclusion plots we have just discussed. Our excluded regions depend on parameters which suffer from some uncertainties: in particular the (unscaled) value for $\rho_{x}$ has been taken at the central value of its standard range $(0.2-$ $0.4) \mathrm{GeV} \mathrm{cm}^{-3}$ and the coupling of Higgs scalars to hadrons (which represent the dominant contribution to the capture rate) could be somewhat overes- timated ${ }^{\sharp 4}$. A modification in the values for these parameters would relax the experimental constraints accordingly.

We also wish to emphasize that our exclusion plots have been derived by rescaling $\rho_{x}$ by the factor $\Omega h^{2} /$ 0.05 whenever $\Omega h^{2}$ is less than 0.05 [18]. Indeed, when the neutralino relic abundance $\Omega h^{2}$ is less than 0.05 , neutralinos cannot provide by themselves the total amount of invisible matter of the galactic halo. Derivations of exclusion plots which do not take into account the actual $\Omega h^{2}$ values and so would give much larger excluded regions in the $M_{2}-\mu$ plane are not theoretically consistent.

To illustrate the capabilities of investigation by larger neutrino telescopes in our figures we also give the regions which could be explored by a detector of the size of $10^{5} \mathrm{~m}^{2}$, with a muon energy threshold of 2 $\mathrm{GeV}$, in two years of running; the dotted domains indicate where the signal would give a 4- $\sigma$ effect under those conditions. Also reported in fig. 2 are the regions excluded by LEP [39]. A few comments are in order here: (i) radiative corrections to the Higgs masses modify the size of the muon signal significantly (this is particularly noticeable for negative values of $\mu$ and at low $\tan \beta$ ): (ii) neutrino telescopes are a very useful tool to investigate the MSSM theory in parameter regions which are complementary to those explored by accelerators.

We wish to thank V.S. Berezinsky, G. Gelmini, P. Gondolo, G. Ridolfi, D. Seckel and F. Zwirner for useful discussions. This work was supported in part by the Research Funds of the Ministero dell' Università $\mathrm{e}$ della Ricerca Scientifica e Tecnologica.

\#4 For a new evaluation of the pion-nucleon $\sigma$-term which implies a coupling of the Higgs scalars to hadrons which is smaller than that given in refs. [31.32], see ref. [41].

\section{References}

[1] M. Spiro, in: Proc. Third Intern. Workshop on Neutrino telescopes (Venice. 1991), ed. M. Baldo Ceolin, to be published.

[2] E.A. Valentijn, Nature 346 (1990) 153.

[3] W. Saunders ct al., Nature 349 (1991) 32.

[4] P. Fayet, Phys. Lett. B 86 (1979) 272. 
[5] K. Griest, Phys. Rev. D 38 (1988) 2357; Phys. Rev. Lett. $61(1988) 666$.

[6] J. Ellis. R.A. Flores and J.D. Lewin, Phys. Lett. B 212 (1988) 375 .

[7] R. Barbieri, M. Frigeni and G.F. Giudice, Nucl. Phys. B 313 (1989) 725 .

[8] R. Flores, K.A. Olive and M. Srednicki, Phys. Lett. B 237 (1990) 72.

[9] E.A. Paschos, A. Pilaftsis and K. Zioutas, Phys. Lett. B 236 (1990) 321.

[10] L.M. Krauss, M. Srednicki and F. Wilczek. Phys. Rev. D 33 (1986) 2079.

[11] M. Srednicki, K.A. Olive and J. Silk, Nucl. Phys. B 279 (1987) 804

[12] J.S. Hagelin, K.W. Ng and K.A. Olive, Phys. Lett. B 180 (1986) 375 .

[13] K.W. Ng, K.A. Olive and M. Srednicki, Phys. Lett. B 188 (1987) 138 .

[14] K.A. Olive and M. Srednicki, Phys. Lett. B 205 (1988) 553.

[15] J. Ellis, R.A. Flores and S. Ritz, Phys. Lett. B 198 (1987) 393.

[16] S. Ritz and D. Seckel, Nucl. Phys. B 304 (1988) 877.

[17] G.F. Giudice and E. Roulet, Nucl. Phys. B 316 (1989) 429.

[ 18 ] G.B. Gelmini, P. Gondolo and E. Roulet, Nucl. Phys. B 351 (1991) 623.

[19] J. Ellis, Invited talk Nobel Symp. on Early cosmology (Graftavallen, June 1990), preprint CERN-TH 5822/90, and references therein.

[20] P. Fayet, in: Unification of the fundamental particle interactions, eds. S. Ferrara, J. Ellis and P. van Nieuwenhuizen (Plenum, New York, 1980) p. 587; H.E. Haber and G.L. Kane, Phys. Rep. 117 (1985) 75; J.F. Gunion and H.E. Haber, Nucl. Phys. B 272 (1986) l: R. Barbieri, Riv. Nuovo Cimento 11 (1988) 4.

[21] J. Ellis, R.A. Flores, K. Freese, S. Ritz, D. Seckel and J. Silk, Phys. Lelt. B 214 ( 1988 ) 403;

L. Bergstrom, Nucl. Phys. B 325 (1989) 647:

K. Freese and J. Silk, Phys. Rev. D 40 (1989) 3828:

H. Bengtsson, P. Salati and J. Silk, preperint CERN-TH5639/90;

S. Rudaz and F. Stecker, University of Minnesota preprint UMN-TH 823/90.

[22] J. Ellis, G. Ridolvi and F. Zwirner, preprint CERN-TH 5946/90 (1990):

H. Haber and R. Hempfling, preprint SCIPP-90/42 (1990): Y. Okada, M. Yamaguchi and T. Yanagida, preprint TU$360(1990)$ :

R. Barbieri, M. Frigeni and F. Caravaglios, preprint IFUPTH 46/90 (1990):

R. Barbieri and R. Frigeni, preprint IFUP-TH 2/91 (1991).
[23] J. Ellis, J.S. Hagelin, D.V. Nanopoulos, K. Olive and $M$ Srednicki, Nucl. Phys. B 238 (1984) 453.

[24] M. Srednicki, R. Watkins and K.A. Olive, Nucl. Phys. B 310 (1988) 693.

[25] J. Ellis, L. Roszkowski and Z. Lalak, Phys. Lett. B 245 (1990) 545

[26] J. Ellis, D.V. Nanopoulos, L. Roszkowski and D.N. Schramm, preprint CERN-TH-5705/90.

[27] ALEPH Collab., D. Decamp et al., Phys. Lett. B 241 (1990) 141;

J.F. Grivaz, preprint ALEPH 90-184:

L. Rolandi, private communication;

DELPHI Collab., P. Abreu ct al., preprint CERN-PPE/90163 (1990):

L3 Collab., B. Adeva et al., Phys. Lett. B 251 (1990) 311; OPAL Collab., M.Z. Akrawy et al., preprint CERN-PPE/ 90-100 ( 1990$)$.

[28] A. Gould, Astrophys. J. 321 (1987) 561

[29] A. Gould, Astrophy's. J. 321 (1987) 571: see also A. Gould, Astrophys. J. 328 (1988) 919.

[30] W.H. Press and D.N. Spergel. Astrophys. J. 294 (1985) 663; 296 (1985) 679, and references therein.

[31] T.P. Cheng, Phys. Rev. D 38 (1988) 2869.

[32] H.Y. Cheng, Phys. Lett. B 219 (1989) 347.

[33] T.K. Gaisser and T. Stanev, Phys. Rev. D 30 (1984) 985.

[34] V.S. Berezinsky, S.V. Bulanov, V.A. Dogiel, V.L. Ginzburg and V.S. Ptuskin, Astrophysics of cosmic rays (Elsevier, Amsterdam, 1990), and references therein.

[35] Particle Data Group, J.J. Hernández et al., Review of particle properties, Phys. Lett. B 239 (1990) III.13; W. Lohmann, R. Kopp and R. Voss, CERN report 85-03 (1985).

[36] Particle Data Group. C.G. Wohl et al., Rev. Mod. Phys. 56 (1984) S61.

[37] L.V. Volkova. Sov. J. Nucl. Phys. 31 (1980) 784.

[38] KAMIOKANDE Il Collab., K.S. Hirata et al., presented by M. Mori at 3rd Intern. Workshop on Neutrino telescopes (Venice, 1991).

[39] ALEPH Collab., D. Decamp et al.. Phys. Lett. B 244 (1990) 541:

J. Ellis, G. Ridolfi and F. Zwirner, Phys. Lett. B 237 (1990) 423:

F. Zwirner, private communication.

[40] Frejus Collab., Ch. Berger et al., Z. Phys. C 48 (1990) 221; L. Moscoso, Proc. Intern. Workshop on Neutrino telescopes, ed. M. Baldo Ceolin (1988);

IMB Collab., J.M. Losecco et al., Phys. Lett. B 188 (1987) 388;

NUSEX Collab., M. Aglietta et al., to be published.

[41] J. Gasser, H. Leutwyler and M.E. Sainio. Phys. Lett. B 253 (1991) 252. 